\title{
Electrochemical reduction of azo dyes mimicking their biotransformation to more toxic products
}

\author{
Konrad Pietruk, Marta Piątkowska, Małgorzata Olejnik \\ Department of Pharmacology and Toxicology, \\ National Veterinary Research Institute, 24-100 Puławy, Poland \\ konrad.pietruk@piwet.pulawy.pl
}

Received: January 25, 2019

Accepted: July 2, 2019

\begin{abstract}
Introduction: Some azo dyes, including Sudans I-IV and Para Red, are genotoxic and may be biotransformed to cancerogenic aromatic amines. They are banned as food and feed additives, but their presence has been detected in food. Aromatic amines are also considered potentially toxic. Online EC-MS is a promising tool to study the transformation mechanisms of xenobiotics such as azo dyes. The aim of the study was to investigate emulation of how azo dyes are enzymatically transformed to amines with EC-MS. Material and Methods: The reduction reactions of five azo dyes (Sudans I-IV and Para Red) were conducted using a glassy carbon working electrode and $0.1 \%$ formic acid in acetonitrile. Reduction results were compared with the literature and in silico to select preliminary candidates for metabolites. The LC-MS/MS method was used to confirm results obtained by electrochemical reactor. Results: A limited number of pre-selected compounds were confirmed as azo dyes metabolites - aniline for Sudan I, aniline and 4-aminoazobenzene for Sudan III, $o$-toluidine for Sudan IV, and 4-nitroaniline for Para Red. No metabolites were found for Sudan II. Conclusions: Electrochemistry-mass spectrometry was successfully applied to azo dyes. This approach may be used to mimic the metabolism of azo dyes, and therefore predict products of biotransformation.
\end{abstract}

Keywords: azo dyes, red-ox, biomimetics, 3R, replacement.

\section{Introduction}

Sudan I-IV dyes have been recognised by the International Agency for Research on Cancer (IARC) as group 3 carcinogenic agents, potentially carcinogenic to humans, due to insufficient data on their carcinogenicity to animals to recommend a higher risk group designation (11). Para Red has not been classified by the IARC; however, it is structurally very similar to Sudan I and therefore it is suspected to exert equally toxic effects. Although these dyes are forbidden to be used as food and feed additives, their presence is continuously detected in spices, these mainly being chilli, curry or curcuma (18).

The mechanism of carcinogenicity of azo dyes is related to metabolic activation to produce reactive intermediates that can bind to DNA. The main mechanism is considered a reductive cleavage of the azo linkage to release aromatic amines (2). Therefore the toxicity of azo dyes would be closely related to corresponding amines present in an azo dye structure (1).
As reported in the literature, the dyes selected for the experiment undergo enzymatic reactions in the human body and are transformed into aromatic amines: Sudans I and III into aniline, Sudan II into 2,4dimethylaniline, Sudan IV into $o$-toluidine, and Para Red into $p$-nitroaniline (22).

Despite the ban on azo dyes, there is still a risk of their occurrence in food. As shown by Chen et al. (5), azo dyes were found in eggs and animal tissues intended for human consumption. It might result from their presence in oleoresins used as chicken feed additives to enhance egg yolk colour (10). However, the previous studies performed in our department indicated that the transfer of Sudan I to eggs was very low (16). Additionally, the amount of the dye present in the gastrointestinal tract was lower than its initial amount in the prepared experimental feed, which was indirect evidence of its transformation in the gastrointestinal tract of laying hens (16).

The combination of electrochemistry with mass 
spectrometry has recently become a popular approach to simulating metabolisation of xenobiotics (4). Quick and easy screening for possible reaction products make this technique particularly promising in metabolic studies $(3,7,12,19)$.

Therefore the aim of this study was to investigate the reduction products of selected azo dyes using the technique of electrochemistry with mass spectrometry (EC-MS). Afterwards, a comparison was made with data available in literature and software for predicting metabolic pathways. Particular attention was paid to the harmful aromatic amine reduction EC products. Based on these results an analytical method using liquid chromatography combined with tandem mass spectrometry (LC-MS/MS) was developed as a tool for the confirmation of the identified products obtained by electrochemical reduction.

\section{Material and Methods}

Chemicals and reagents. Acetonitrile and 99.5\% formic acid (all HPLC grade) were provided by J.T. Baker (USA). Ammonium acetate, acetone, analytical standards of Sudan I-IV dyes, Para red, and their metabolites were purchased from Sigma-Aldrich (Germany). Water was purified through a Milli-Q plus system from Millipore (USA).

The stock standard solutions $(1,000 \mu \mathrm{g} / \mathrm{ml})$ of Sudan I and Sudan II were prepared by weighing appropriate amounts of the substances and dissolving Sudan III, Sudan IV, and Para Red in acetonitrile, 4,4'-oxydianiline in acetone, 4-aminoazobenzene, 2,4-dimethylaniline, aniline and 4-nitroaniline in methanol, and 1-amino-2-naphthol in water. All stock solutions were kept in the dark below $-18^{\circ} \mathrm{C}$ for six months. A solution of an internal standard was prepared separately.

Working standard solutions of the dyes were prepared by dissolving appropriate amounts of stock standard solutions in acetonitrile and they were kept in the dark at $4-6^{\circ} \mathrm{C}$ for three months. Working standard solutions of metabolites were prepared by dissolving appropriate amounts of stock standard solutions in water before each analysis.

Electrochemical and mass-spectrometrical simulation (EC-MS). The ROXY electrochemical (EC) analyser (Antec, the Netherlands), was equipped with a ReactorCell infusion pump connected to an LC-MS 8050 triple-quadrupole mass spectrometer (Shimadzu, Japan). The reduction reactions were performed in an electrochemical reactor cell equipped with glassy carbon as a working electrode, HyREF as a reference electrode $(\mathrm{Ag} / \mathrm{AgCl})$, and a platinum auxiliary electrode. The tested dye solutions at the concentration of $10 \mu \mathrm{g} / \mathrm{mL}$ in $0.1 \%$ formic acid in acetonitrile were infused to the experimental cell under a constant flow of $10 \mu \mathrm{L} / \mathrm{min}$ using a syringe pump. The electrolysis experiment was conducted using the scan mode from $-2 \mathrm{~V}$ to $0 \mathrm{~V}$ at the rate of $10 \mathrm{mV} / \mathrm{s}$. The products of the electrochemical reaction were analysed by mass spectrometer working in the selected ion monitoring mode of $\mathrm{m} / \mathrm{z}$ ratio for each analysed azo dye and in full scan mode to acquire the $\mathrm{m} / \mathrm{z}$ ratio of metabolites formed from each azo dye.

For confirmation analysis, an electrochemical reactor was used working in offline mode with conditions set as described above, except voltage which was set to $-2 \mathrm{~V}$. About $1 \mathrm{~mL}$ of reduction products for each azo dye was collected and analysed using the LC-MS/MS method.

Data interpretation of the results from electrochemical simulation. Data from extracted ion chromatograms were used to acquire the masses of potential metabolites of each azo dye. These results were matched with data found in literature regarding metabolisation of Sudans I-IV and Para Red, and an in silico study was conducted using available software: EAWAG-BBD Pathway Prediction System (20) and BioTransformer (9).

Chromatography and mass spectrometry of analytical method for identification of potential metabolites. The potential candidates were finally verified by developing an analytical method using LC/MS-MS technique. The LC-MS/MS system consisted of a Nexera X2 UHPLC liquid chromatograph (Shimadzu, Japan) coupled with an 8050 triple quadruple detector (Shimadzu) used in positive and negative mode with ESI and controlled by LabSolutions 5.60 SP2 software (Shimadzu). The parameters were set as follows: nebulising gas (nitrogen) flow $-2 \mathrm{~L} / \mathrm{min}$, heating and drying gas (air) flow, each $10 \mathrm{~L} / \mathrm{min}$, interface temperature at $300^{\circ} \mathrm{C}$, desolvation line temperature at $250^{\circ} \mathrm{C}$, heat block temperature at $400^{\circ} \mathrm{C}$, and capillary voltage at -3 and $4 \mathrm{kV}$ for negative and positive ionisation, respectively. The mass analyser was configured in selected reaction monitoring mode; each analyte was analysed using two transitions. The compounds were separated using a Kinetex F5 (100 mm, $2.1 \mathrm{~mm}, 2.6 \mu \mathrm{m}$ ) analytical column (Phenomenex, USA) and acetonitrile (A) and $10 \mathrm{mM}$ ammonium acetate $\mathrm{pH}$ 7.0 (B) as a mobile phase. The following gradient elution was applied in a $10-\mathrm{min}$ run time: $30 \% \mathrm{~B}$ from 0 to $1.2 \mathrm{~min}$, increasing to $95 \% \mathrm{~B}$ until the $2.0 \mathrm{~min}$ time, this was maintained until the $8.0 \mathrm{~min}$ point, and then the column was re-equilibrated to the initial condition from 8.2 to $10 \mathrm{~min}$.

\section{Results}

Electrochemical simulation. To obtain the highest efficiency of the reduction of azo dyes the following parameters were optimised: the concentration of formic acid, concentration of azo dyes used in the solution for reduction reactions, and the material from which the working electrode was made.

The first step to establish the optimal conditions for electrochemical reduction was to set the concentration 
of formic acid in acetonitrile. Therefore, azo dyes (at the concentration of $10 \mu \mathrm{g} / \mathrm{mL}$ ) were prepared in the following solutions: $0.01 \%, 0.05 \%, 0.1 \%$, and $0.5 \%$ of formic acid with acetonitrile. The highest signals of reduction products were observed for the $0.1 \%$ and $0.5 \%$ solutions. For the next step of optimisation, the $0.1 \%$ formic acid solution was chosen. Afterwards, the influence of the concentration of the azo dyes was studied, and the following concentrations were prepared and analysed: 2, 5, 10, 20, and $40 \mu \mathrm{g} / \mathrm{mL}$. Below
$5 \mu \mathrm{g} / \mathrm{mL}$, none of the electrochemical products were observed. Concentration of $10 \mu \mathrm{g} / \mathrm{mL}$ was selected as optimal, because higher concentrations did not provide meaningfully higher signals of reduction products. Finally, the performance of the electrochemical reduction was compared using the two different working electrodes of glassy carbon and gold. Significantly higher signals were obtained using glassy carbon as a working electrode.

Table 1. Signals ( $\mathrm{m} / \mathrm{z}$ ratio) acquired during the electrochemical step

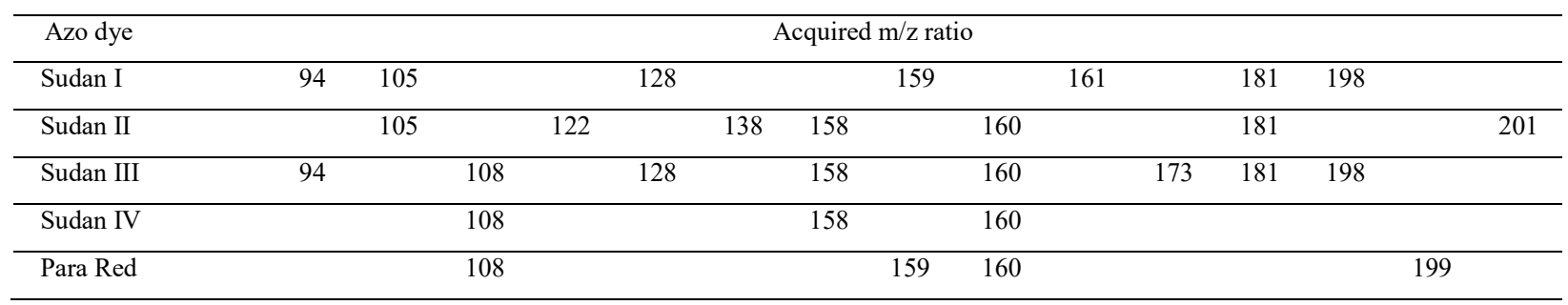

Table 2. Compounds selected as the candidates for metabolites of azo dyes selected from the literature and in silico data

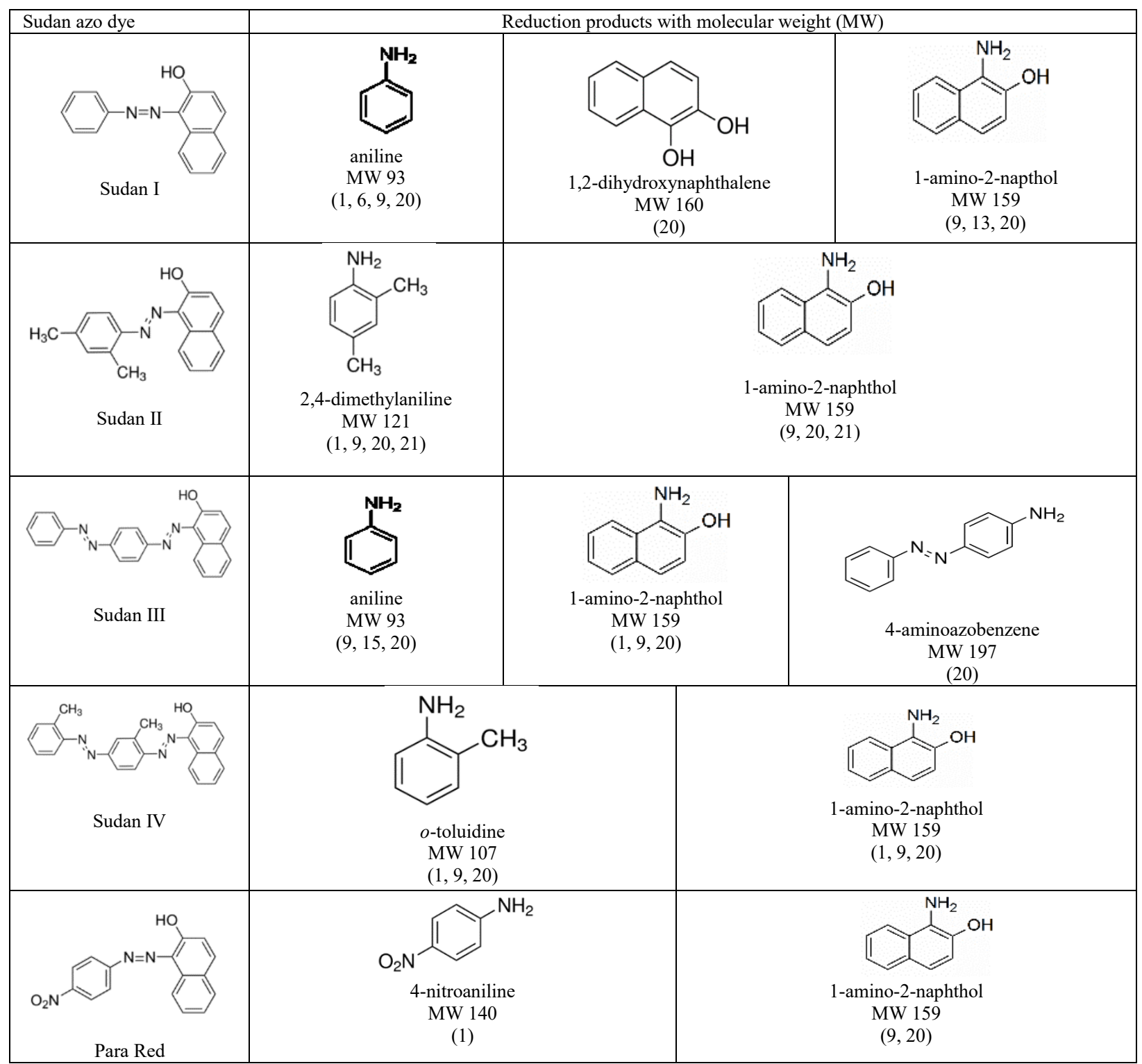


Table 3. Mass spectrometry parameters for analytes in scope of the LC-MS/MS method

\begin{tabular}{lllll}
\hline Analyte & Ionisation & Precursor & Products & Collision energy $(\mathrm{eV})$ \\
\hline 1,2-dihydroxynaphthalene & positive & 161.0 & $131.0 / 77.1$ & $-33.0 /-18.0$ \\
\hline 2,4-dimethyaniline & positive & 122.6 & $108.1 / 80.1$ & $-27.0 /-21.0$ \\
\hline 1-amino-2-naphthol & positive & 160.0 & $130.0 / 103.3$ & $-13.0 /-11.0$ \\
\hline 4-aminoazobenzene & positive & 197.6 & $93.2 / 77.1$ & $-25.0 /-49.0$ \\
\hline 4-nitroaniline & negative & 137.0 & $107.0 / 93.0$ & $30.0 / 18.0$ \\
\hline aniline & positive & 94.2 & $77.1 / 51.0$ & $-25.0 /-30.0$ \\
\hline$o$-toluidine & positive & 108.0 & $91.1 / 65.1$ & $-21.0 /-27.0$ \\
\hline
\end{tabular}

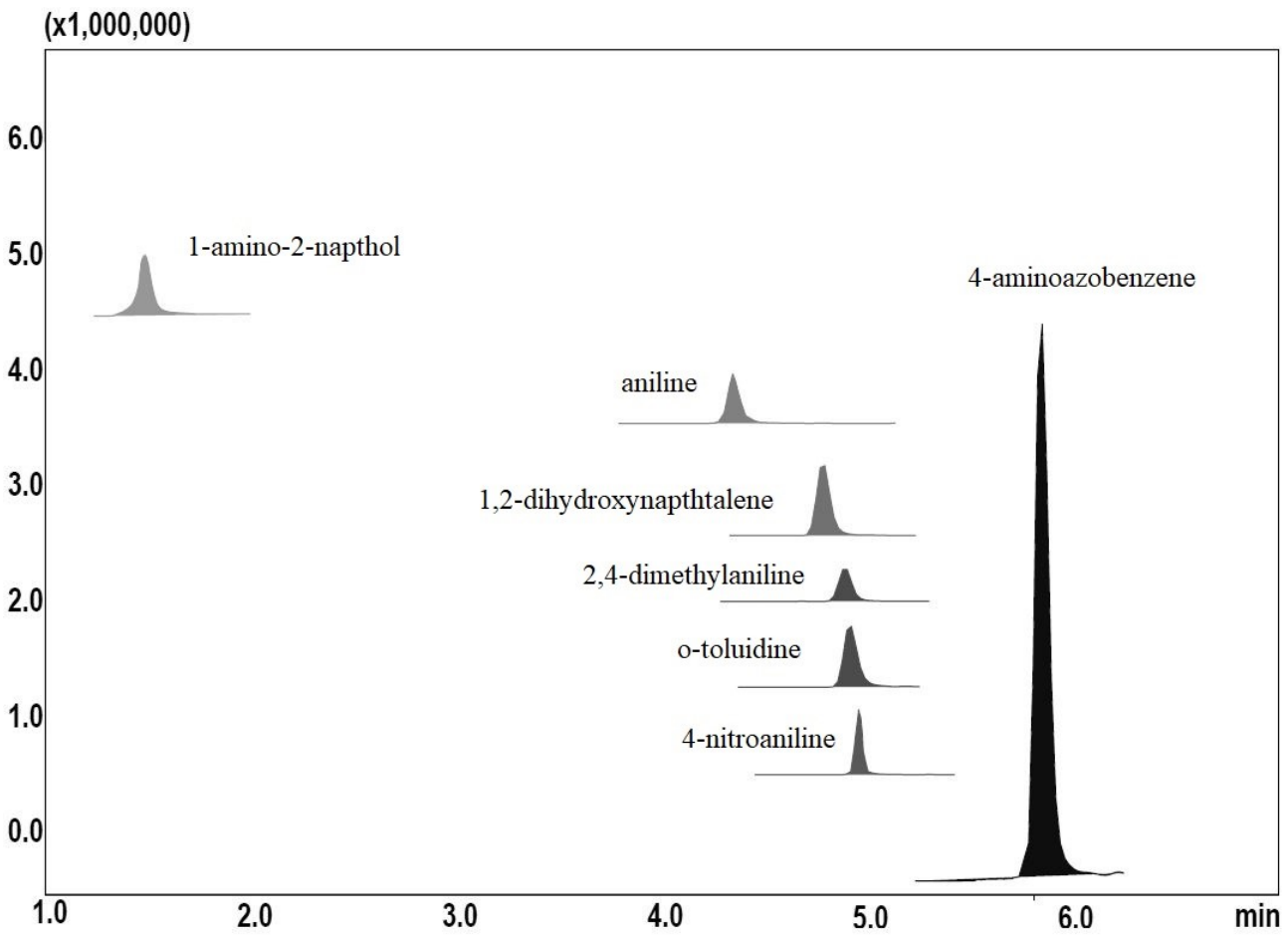

Fig. 1. Chromatogram of candidates for metabolites at concentration of $1 \mu \mathrm{g} / \mathrm{mL}$

On the basis of the optimisation experiments the following conditions were used: $0.1 \%$ concentration of formic acid, $10.0 \mu \mathrm{g} / \mathrm{mL}$ concentration of azo dyes in the solution, and glassy carbon as a working electrode.

All the selected dyes successfully underwent the reduction reactions using the optimised conditions, resulting in a wide range of electrochemical signals. The acquired $\mathrm{m} / \mathrm{z}$ ratios of signals obtained for all azo dyes are presented in Table 1. This approach produced a large number of signals; therefore interpretation of the data was required to designate the most promising candidates for metabolites of analysed azo dyes.

Data interpretation of the electrochemical simulation step. The preliminary identification of signals obtained in electrochemical simulation was performed by comparing our results with the data in the literature. As an additional identification method we applied an in silico approach by using web-based software to predict plausible pathways for degradation of chemical compounds $(9,20)$. The predictions using the EAWAG-BBD Pathway Prediction System and BioTransformer (in which predictions were performed using the Human Gut Microbial option) were similar, but some discrepancies were observed. Sudan I to 1,2-dihydroxynapthalene and Sudan III to 4-aminoazobenzene were predicted only by EAWAGBBD software.

Chromatography and tandem mass spectrometry analytical method for the identification of potential metabolites. To confirm our findings from previous steps of the experiment, an analytical method using an LC-MS/MS technique was developed. The MS/MS fragmentation conditions of compounds identified in previous steps were investigated and collision energies for each individual compound were optimised to give sufficient sensitivity. Pseudomolecular ions were used for all analytes as a precursor and two product ions were monitored. The detection of all compounds was conducted using positive ionisation, except 4-nitroaniline which was monitored in negative 
ionisation. The MS/MS conditions are summarised in Table 3.

The Phenomenex Kinetex F5 column combined with the gradient profile of ammonium acetate and acetonitrile gave satisfactory peak shapes and separation of all the analytes. The chromatogram of the standard solution of metabolite candidates at the concentration of $1 \mu \mathrm{g} / \mathrm{mL}$ is shown in Fig. 1.

The developed method was then used to analyse solutions produced during the offline electrochemical simulation step. Only a limited number of pre-selected metabolites were confirmed. For Sudan I only aniline was found, while for Sudan II no analytes were detected. Analysis of the Sudan III reduction solution confirmed the presence of both aniline and 4-aminoazobenzene. The presence of $o$-toluidine was confirmed in the Sudan IV solution and in that of Para Red only the presence of 4-nitroaniline was noted.

\section{Discussion}

Azo dyes are diazotised amines coupled to an amine or phenol, with one or more azo bonds $(-\mathrm{N}=\mathrm{N}-)$. The dyes selected for the tests (Sudans I-IV and Para red) are banned for use in food and feed, nevertheless their presence is still detected in spices and foods which contain them, eggs, and animal tissues (6). Recent research on natural contamination has demonstrated that the combination of Sudan I-contaminated soils and application of Sudan I-containing agricultural preparations constitutes a major source of Sudan I in vegetables during the growth period, at the levels of $0.18-2.52 \mu \mathrm{g} / \mathrm{kg}(13)$. Usually azo dyes are resistant to biodegradation, but there are chemical and biological systems capable of reducing them. Sudan dyes may be transformed by anaerobic bacteria in soil, sludge, and the intestines into carcinogenic aromatic amines (22), which exert more toxic health effects than the dyes themselves. Thus we decided to study this phenomenon using abiotic methods, which allows the possible metabolites of Sudan azo dyes in animal and human bodies to be predicted.

The results of the previously published experiments regarding the reduction of azo dyes to aromatic amines are very diverse, and the range of analytical techniques applied in these studies is very wide.

For Sudan I, aniline was identified by other authors as the main metabolite of anaerobic degradation $(21,22)$.

Additionally, the presence of 1-amino-2-naphthol as a metabolite of Sudan I was expected in another publication (15). In current studies only the presence of aniline as a Sudan I metabolite was confirmed by the LC-MS/MS system using analytical standards.

The studies of Sudan II conducted by Yaguez et al. (8) revealed that the electrochemical reaction involves a four-electron exchange in both acidic and basic media, resulting in the formation of 1-amino-2-naphthol and 2,4-dimethyl-aniline; 2,4-dimethyl-aniline was also identified as the metabolite produced from Sudan II by E. faecalis (22). In the present studies we were not able to confirm any of the pre-selected metabolites.

Pielesz et al. (17) reduced Sudan III using sodium hydrosulphite in an alkaline medium and reported aniline and $p$-phenylenediamine as main products. Other authors used sodium thiosulphate and performed electrochemical reduction measurements on Sudan III to report the formation of aniline, benzidine, $o$-toluidine, 2,6-dimethylaniline, 4,4'-oxydianiline, and 4,4'methylene-bis(2-methylaniline) using the HPLC-DAD detection system (14).

In the studies on biodegradation of Sudan IV proposed by Xu et al. (22) the metabolic pathway led to 1-amino-2-napthol, 2,5-diaminotoluene and $o$-toluidine. The chemical structure of Para Red is similar to Sudan I therefore similar properties are expected. For Para red reduction by $E$. faecalis to 4-nitroaniline was identified in the literature, and in other studies reduction to 1,2-naphthoquinone, 4,4'-diaminodiphenylmethane, and 1-(2-hydroxyfenyloazo)-2-naphthol was established (22). In our studies only the 4-nitroaniline metabolite was confirmed, to do which the LC/MS-MS technique was used.

The electrochemical reactor combined with mass spectrometer was successfully applied to simulate the reduction of five azo dyes. One constraining factor in this research was that only limited compounds preselected as the metabolites of azo dyes were afterwards confirmed by the LC-MS/MS method.

Conflict of Interests Statement: The authors declare that there is no conflict of interests regarding the publication of the article.

Animal Rights Statement: None required.

Finance Disclosure Statement: This study was financed by the National Science Centre Poland, through the PRELUDIUM 9 funding competition, under award No 2015/17/N/NZ7/04097: in simulacra studies on the reduction of azo dyes and identification of their carcinogenic metabolites.

Acknowledgements: Sincere thanks to SHIM-POL for sharing the ROXY EC/MS-dedicated electrochemistry system (Antec Scientific) and organising training, which enabled the studies to be conducted.

\section{References}

1. Bartsch H.: Metabolic activation of aromatic amines and azo dyes. IARC Sci Publ 1981, 40, 13-30.

2. Brown M.A., De Vito S.C.: Predicting azo dye toxicity. Crit Rev Environ Sci Technol 1993, 23, 249-324.

3. Bruins A.P.: An overview of electrochemistry combined with mass spectrometry. TrAC Trends Anal Chem 2015, 70, 14-19.

4. Bussy U., Delaforge M., El-Bekkali C., Ferchaud-Roucher V., Krempf M., Tea I., Galland N., Jacquemin D., Boujtita M.: Acebutolol and alprenolol metabolism predictions: Comparative 
study of electrochemical and cytochrome P450-catalyzed reactions using liquid chromatography coupled to high-resolution mass spectrometry. Anal Bioanal Chem 2013, 405, 6077-6085.

5. Chen D., Cao X., Tao Y., Wu Q., Pan Y., Peng D., Liu Z., Huang L., Wang Y., Wang X., Yuan Z.: Development of a liquid chromatography-tandem mass spectrometry with ultrasoundassisted extraction and auto solid-phase clean-up method for the determination of Fusarium toxins in animal derived foods. J Chromatogr A 2013, 1311, 21-29.

6. Chen D., Li X., Tao Y., Pan Y., Wu Q., Liu Z., Peng D., Wang X., Huang L., Wang Y., Yuan Z.: Development of a liquid chromatography-tandem mass spectrometry with ultrasoundassisted extraction method for the simultaneous determination of sudan dyes and their metabolites in the edible tissues and eggs of food-producing animals. J Chromatogr B Anal Technol Biomed Life Sci 2013, 939, 45-50.

7. Chen L., Hofmann D., Klumpp E., Xiang X., Chen Y., Küppers S.: Bottom-up approach for the reaction of xenobiotics and their metabolites with model substances for natural organic matter by electrochemistry-mass spectrometry (EC-MS). Chemosphere 2012, 89, 1376-1383.

8. de la Cruz Yaguez L.I., Pingarron Carrazon J.M., Polo Diez L.M. Polarographic study of the 1-(2,4-dimethylphenylazo)-2-naphthol (Sudan II) in hydroalcoholic medium. Electrochim Acta 1986, 31, 119-121.

9. Djoumbou-Feunang Y., Fiamoncini J., Gil de la Fuente A., Greiner R., Manach C., Wishart D.S.: BioTransformer: a comprehensive computational tool for small molecule metabolism prediction and metabolite identification. J Cheminform 2019, 11, $1-25$.

10. Hoenicke K.: AOAC Europe Section - International Workshop "Foods to Dye For." 2006, 1-20.

11. IARC: Overall Evaluations of Carcinogenicity: An Updating of IARC monographs volumes 1 to 4. IARC Monogr Eval Carcinogenic Risks to Humans 1987, 1-42.

12. Karst U.: Electrochemistry/Mass Spectrometry (EC/MS) A New Tool To Study Drug Metabolism and Reaction Mechanisms. Angew Chemie Int Ed 2004, 43, 2476-2478.
13. Lian Y., Gao W., Zhou L., Wu N., Lu Q., Han W., Tie X. Occurrence of Sudan I in Paprika Fruits Caused by Agricultural Environmental Contamination. J Agric Food Chem 2014, 62 , 4072-4076.

14. Lizier T.M., Zanoni T.B., de Oliveira D.P., Zanoni M.V.B.: Electrochemical reduction as a powerful tool to highlight the possible formation of by-products more toxic than Sudan III dye. Int J Electrochem Sci 2012, 7, 7784-7796.

15. Møller P., Wallin H.: Genotoxic hazards of azo pigments and other colorants related to 1-phenylazo-2-hydroxynaphthalene. Mutat Res 2000, 462, 13-30.

16. Piątkowska M., Jedziniak P., Olejnik M., Żmudzki J., Posyniak A.: Absence of evidence or evidence of absence? A transfer and depletion study of Sudan I in eggs. Food Chem 2018, 239, 598-602.

17. Pielesz A., Baranowska I., Rybak A., Włochowicz A.: Detection and determination of aromatic amines as products of reductive splitting from selected azo dyes. Ecotoxicol Environ Saf 2002, 53, 42-47.

18. RASFF: Annual report on the functioning of the RASFF. Ann Rep 2003, 1-32.

19. Szultka-Młynska M., Buszewski B.: Electrochemistry-mass spectrometry for in-vitro determination of selected chemotherapeutics and their electrochemical products in comparison to in-vivo approach. Talanta 2016, 160, 694-703.

20. Wicker J., Fenner K., Ellis L., Wackett L., Kramer S.: Predicting biodegradation products and pathways: A hybrid knowledge- and machine learning-based approach. Bioinformatics 2010, 26, 814-821.

21. Xu H., Heinze T.M., Chen S., Cerniglia C.E., Chen H.: Anaerobic metabolism of 1-amino-2-naphthol-based azo dyes (Sudan dyes) by human intestinal microflora. Appl Environ Microbiol 2007, 73, 7759-7762.

22. Xu H., Heinze T.M., Paine D.D., Cerniglia C.E., Chen H.: Sudan azo dyes and Para Red degradation by prevalent bacteria of the human gastrointestinal tract. Anaerobe 2010, 16, 114-119. 